\title{
Integral equation and simulation studies of a realistic model for liquid hydrogen chloride
}

\author{
C. Martín, E. Lomba, and M. Lombardero \\ Instituto de Quimica Fisica Rocasolano, CSIC, Serrano 119, E-28006 Madrid, and Departamento \\ Química Física I, U. Complutense, E-28040 Madrid, Spain \\ F. Lado \\ Department of Physics, North Carolina State University, Raleigh, North Carolina 27695-8202 \\ J. S. Høye \\ Institutt for Fysikk, Universitetet $i$ Trondheim, N-7034 Trondheim-NTH, Norway
}

(Received 17 August 1993; accepted 6 October 1993)

\begin{abstract}
Liquid hydrogen chloride is modeled by a system of heteronuclear two-center Lennard-Jones particles with embedded point dipoles and quadrupoles. The effect of molecular polarizability is incorporated via an effective dipole approximation. The study is performed by Monte Carlo reaction field simulation and by hypernetted chain and reference hypernetted chain integral equations. Our simulation results yield dielectric properties in excellent agreement with experimental data for liquid $\mathrm{HCl}$. As for the integral equation approach, we have experimented with an empirical choice of the reference system in the spirit of a recently proposed treatment which has proved extremely successful for pure and quadrupolar two-center Lennard-Jones fluids. The hypernetted chain equation performs slightly better when accounting for the multipolar contributions to the configurational energy, but as a whole the reference hypernetted chain equation, as introduced, here proves to be a more appropriatc choice.
\end{abstract}

\section{INTRODUCTION}

Liquid hydrogen chloride has been the focus of numerous computer simulation studies in the past. ${ }^{1-5}$ Several of these works deal with the calculation of dielectric properties of this polar liquid, both by direct evaluation of the Kirkwood factor ${ }^{4}$ and by a perturbation and difference method. ${ }^{3}$ However, as far as we know, the effects of polarizability have not been explicitly taken into account as yet. Here we intend to apply a recently proposed perturbative approach to incorporate fluctuating polarizability in the treatment of classical fluids ${ }^{6}$ by means of an effective dipole moment. The approach is closely connected to the selfconsistent mean field (SCMF) approximation of Patey, Levesque, and Weis, ${ }^{7}$ which has been successfully applied to polar-polarizable liquids like water ${ }^{7}$ and ammonia. ${ }^{8}$ This is, nonetheless, the first time this sort of approach is applied to a model in which the molecular shape is treated explicitly. Here, we have considered liquid hydrogen chloride to consist of heteronuclear two-center Lennard-Jones particles with a point dipole and a linear point quadrupole located at the center of mass. The magnitude of the effective dipole moment is determined as a function of the polarizability and the thermodynamic state via the aforementioned perturbative approach.

Once the polarizable fluid has been reduced to an effective polar fluid problem, one can resort to either simulation or integral equation theories to analyze the properties of the system. We have carried out tedious Monte Carlo simulations using the reaction field method to deal with the effect of the long range of the dipolar forces, first to estimate the magnitude of the effective dipole moment, and then to determine thermodynamics and, very espe- cially, the dielectric constant via direct evaluation of the Kirkwood factor. At the same time, we have solved both the hypernetted chain (HNC) and the reference hypernctted chain (RHNC) integral equations for this system. Although there is an abundant literature concerning the use of these approximations in polar and nonpolar fluids, ${ }^{9-14}$ we are unaware of any calculation involving a soft heteronuclear molecular model.

The definition of the reference system in the RHNC scheme is somewhat problematic. Use of strict optimization procedures is simply ruled out by the large number of parameters involved. Here, to be consistent with an approximation (hereinafter named RHNC-VM) which has proven successful in the case of homonuclear two-center LJ fluids ${ }^{13}$ even with quadrupoles added, ${ }^{14}$ the reference system is a heteronuclear hard diatomic whose properties are calculated using a modified version of an approximation due to Verlet. ${ }^{15}$ Labik and co-workers introduced this modification in the context of homonuclear hard dumbbells $^{16}$ and it was also applied successfully by the same authors to heteronuclear hard dumbbells. ${ }^{17}$ Although in Ref. 13 an efficient empirical criterion for the determination of an appropriate reference system was introduced, the problem in the present instance is slightly more involved. Actually, one has to use an additional adjustable parameter, namely, the ratio between the hard sphere diameters in the heteronuclear hard dumbbell. This implies that in principle it is not possible to treat both interaction sites on the same footing. A general empirical relation that may enable a simple determination of a close-to-optimum reference system from the macroscopic and microscopic parameters 
of the system to be studied is currently under investigation. $^{18}$

This paper is divided into three parts. In Sec. I we define the model and discuss some details of the simulation method and the effective dipole approach. In Sec. II we briefly summarize the leading aspects of the RHNC theory as applied here. And, finally, results and conclusions are given in Sec. III.

\section{THE MODEL. EFFECTIVE DIPOLE APPROACH FOR POLAR/POLARIZABLE FLUIDS}

We consider an $\mathrm{HCl}$ molecule to be a two-center Lennard-Jones diatomic with a permanent dipole $\boldsymbol{\mu}$, a linear quadrupole moment $\mathbf{Q}$, and isotropic polarizability $\alpha$, located at the center of mass. The interaction potential is then given by

$$
\begin{aligned}
\beta u(12)= & 4 \sum_{\alpha, \beta} \frac{\epsilon_{\alpha \beta}}{k T}\left[\left(\frac{\sigma_{\alpha \beta}}{r}\right)^{12}-\left(\frac{\sigma_{\alpha \beta}}{r}\right)^{6}\right]-\frac{\beta \mu^{2}}{r^{3}}\left[3 \frac{\left(\mathbf{s}_{1} \mathbf{r}\right)\left(\mathbf{s}_{2} \mathbf{r}\right)}{r^{2}}-\mathbf{s}_{1} \mathbf{s}_{2}\right]-\frac{3 \beta \mu Q}{r^{4}}\left[\left(\mathbf{s}_{1} \mathbf{r}\right)\left(5 \frac{\left(\mathbf{s}_{2} \mathbf{r}\right)^{2}}{r^{3}}-\frac{1}{r}\right)-\left(\mathbf{s}_{2} \mathbf{r}\right)\left(5 \frac{\left(\mathbf{s}_{1} \mathbf{r}\right)^{2}}{r^{3}}-\frac{1}{r}\right)\right. \\
& \left.-\frac{2}{r}\left(\mathbf{s}_{2} \mathbf{r}-\mathbf{s}_{1} \mathbf{r}\right)\left(\mathbf{s}_{1} \mathbf{s}_{2}\right)\right]-\frac{3 \beta Q^{2}}{4 r^{5}}\left[1-\frac{5}{r^{2}}\left(\mathbf{s}_{1} \mathbf{r}+\mathbf{s}_{2} \mathbf{r}\right)-15 \frac{\left(\mathbf{s}_{1} \mathbf{r}\right)^{2}\left(\mathbf{s}_{2} \mathbf{r}\right)^{2}}{r^{4}}+2\left(\mathbf{s}_{1} \mathbf{s}_{2}-5 \frac{\left(\mathbf{s}_{1} \mathbf{r}\right)\left(\mathbf{s}_{2} \mathbf{r}\right)}{r^{2}}\right)^{2}\right]
\end{aligned}
$$

where $s_{\mathrm{i}}$ is a unit vector describing the orientation of particle $\mathrm{i}, \mathrm{r}$ is the vector joining particles 1 and 2 , and $\sigma_{\alpha \beta}$ and $\epsilon_{\alpha \beta}$ are the Lennard-Jones parameters for each of the sitesite interactions ( $\alpha, \beta$ denote the sites). In Table I we have summarized the molecular parameters used in this work, which are essentially those of model B in Ref. 2 .

According to Ref. 6, a polarizable fluid with reduced dipole moment $\mu^{*}=\mu / \sqrt{\epsilon_{\mathrm{Cl}-\mathrm{Cl}} \sigma_{\mathrm{Cl}-\mathrm{Cl}}^{3}}$ and isotropic polarizability $\alpha^{*}=\alpha / \sigma_{\mathrm{Cl}-\mathrm{Cl}}^{3}$ can be approximated by a pure polar fluid with an effective dipole moment given by

$$
\mu_{\mathrm{eff}}^{* 2}=\frac{3 \alpha^{*} T^{*}}{1+\alpha^{*} c}+\frac{\mu^{* 2}}{1+\alpha^{*} c}
$$

with $T^{*}=k T / \epsilon_{\mathrm{Cl}-\mathrm{Cl}}$ and

$$
c=\left(2 U_{\mathrm{DD}}+U_{\mathrm{DQ}}\right) /\left(N \mu_{\mathrm{eff}}^{* 2} \epsilon_{\mathrm{Cl}-\mathrm{Cl}}\right) .
$$

Here $U_{\mathrm{DD}}$ and $U_{\mathrm{DQ}}$ are the dipole-dipole and the dipolequadrupole contributions to the internal energy using the effective dipole moment.

We have considered two thermodynamic states in the liquid range for which experimental data for the dielectric constant are available: ${ }^{19} T=183.26 \mathrm{~K}$ and $\rho=1.173 \mathrm{~g} / \mathrm{cm}^{3}$ (case A), and $T=258.15 \mathrm{~K}$ and $\rho=0.98 \mathrm{~g} / \mathrm{cm}^{3}$ (case B).

TABLE I. Molecular parameters for liquid hydrogen chloride.

\begin{tabular}{ll}
\hline \hline$\epsilon_{\mathrm{Cl}-\mathrm{Cl}} / k$ & $190.9 \mathrm{~K}$ \\
$\epsilon_{\mathrm{H}-\mathrm{H}} / k$ & $14.7 \mathrm{~K}$ \\
$\sigma_{\mathrm{Cl}-\mathrm{Cl}}$ & $3.47 \AA$ \\
$\sigma_{\mathrm{H}-\mathrm{H}}$ & $2.81 \AA$ \\
$l_{\mathrm{Cl}-\mathrm{H}}$ & $1.30 \AA$ \\
$\alpha$ & $2.64 \AA^{3}$ \\
$\mu$ & $1.08 \mathrm{D}$ \\
$Q$ & $3.93 \mathrm{~B}$ \\
\hline
\end{tabular}

A series of short Monte Carlo runs $\left(5 \times 10^{6}\right.$ configurations for 256 sample particles) were carried out to determine the dipole-dipole and dipole-quadrupole contributions to the internal energy. Using Eq. (2) one can proceed backwards to compute the permanent dipole moment in a polarizable fluid that would yield the effective dipole for which the simulation has been run. In this way, for case A one gets Fig. 1, which shows an almost linear dependence of $\mu_{\text {eff }}^{* 2}$ on $\mu^{* 2}$, at least for the low dipolar strengths considered here. The value of $\mu_{\text {eff }}^{* 2}$ which yields the measured permanent dipole moment is indicated with a filled circle. In Table II we present the values for the internal energy contributions in both cases studied. Note that for the higher temperature case just two points suffice, given the linear behavior found at the lower temperature.

The thermodynamic state parameters in reduced units $\left(T^{*}=k T / \epsilon_{\mathrm{Cl}-\mathrm{Cl}}, \rho^{*}=\rho \sigma_{\mathrm{Cl}-\mathrm{Cl}}^{3}, Q^{* 2}=Q^{2} / \epsilon_{\mathrm{Cl}-\mathrm{Cl} \sigma_{\mathrm{Cl}-\mathrm{Cl}}^{5}}\right)$ together with the calculated effective dipole moments can be found in Table III. The remarkable increase in the dipoledipole interaction induced by the polarizability should be

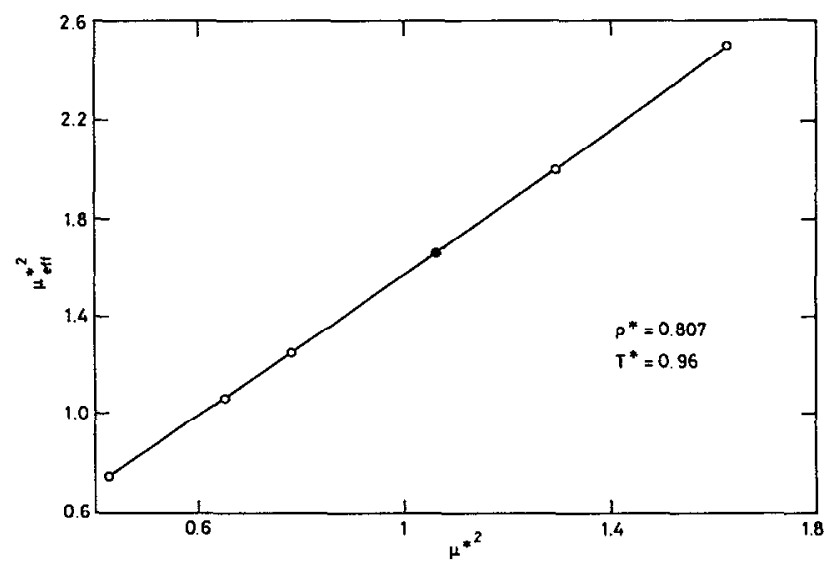

FIG. 1. Effective vs permanent dipole moment for $T=183.2 \mathrm{~K}$ and $\rho=1.173 \mathrm{~g} / \mathrm{cm}^{3}$. 
TABLE II. Dipole-dipole and dipole-quadrupole contributions to the configurational energy as a function of the dipole strength determined from computer simulation.

\begin{tabular}{cccccc}
\hline & \multicolumn{2}{c}{ Case A } & & \multicolumn{2}{c}{ Case B } \\
\cline { 2 - 3 } \cline { 5 - 6 }$\mu_{\mathrm{ec}}^{* 2}$ & $(\beta U / N)_{\mathrm{DD}}$ & $(\beta U / N)_{\mathrm{DQ}}$ & & $(\beta U / N)_{\mathrm{DD}}$ & $(\beta U / N)_{\mathrm{DQ}}$ \\
\hline 0.750 & -0.20 & -1.07 & & -1.38 \\
1.063 & -0.38 & -1.51 & & -0.35 & \\
1.250 & -0.52 & -1.76 & & -1.16 \\
1.659 & & & & -0.52 & \\
2.000 & -1.21 & -2.57 & & \\
2.500 & -1.76 & -3.01 & & \\
\hline \hline
\end{tabular}

noted. The effect of density, although small, is also noticeable; at higher densities one must expect a further increase in the effective values of the dipolar strength due to collective effects.

Once the effective values of the dipolar interaction have been determined, one can proceed to perform a detailed calculation. The determination of the dielectric constant by computer simulation is a rather nettlesome task $^{20,21}$ and there are many problems associated with finite size effects ${ }^{20}$ and the poor convergence of orientational averages. ${ }^{21}$ In order to minimize these problems we have carried out long simulations with relatively large samples, 500 and 864 particles. Long-range interactions were treated using the reaction field method. ${ }^{22,23}$ The number of configurations generated ranges from $2.5 \times 10^{7}$ to $5 \times 10^{7}$. We consider statistical convergence achieved when the orientational averages $\left\langle M^{2}\right\rangle / N \mu^{2}$ and $\left(\left\langle M^{2}\right\rangle-\langle M\rangle^{2}\right) / N \mu^{2}$ yield $g_{K}$ values that differ by less than 1 percent. The evolution of both quantities for the two sample sizes used can be seen in Figs. 2 and 3. The influence of the sample size, although small (and completely negligible as far as thermodynamics and structure are concerned), is still visible, and is also related to the use of larger truncation radii for the largest samples. The results presented below all refer exclusively to 864-particle samples.

The dielectric constant can be evaluated using ${ }^{7}$

$$
f(\epsilon)=\frac{4 \pi}{3} \rho \beta \frac{\left\langle M^{2}\right\rangle}{N}+f\left(\epsilon_{\infty}\right)
$$

where the average $\left\langle M^{2}\right\rangle / N$ includes just the spatial and orientational degrees of freedom, the only ones present in the effective fluid. The function $f(\epsilon)$ depends on the boundary conditions of the simulation and the term $f\left(\epsilon_{\infty}\right)$ accounts for the averaging over the internal degrees

TABLE III. Thermodynamic state parameters for the calculations presented and effective dipoles derived from data of Table II.

\begin{tabular}{lcccccc}
\hline \hline$\rho\left(\mathrm{g} / \mathrm{cm}^{3}\right)$ & $T(K)$ & $\rho^{*}$ & $T^{*}$ & $\mu_{\mathrm{eff}}(\mathrm{D})$ & $\mu_{\mathrm{eff}}^{* 2}$ & $Q^{* 2}$ \\
\hline 1.1734 & 183.26 & 0.80694 & 0.96 & 1.349 & 1.659 & 1.172 \\
0.98 & 258.15 & 0.67394 & 1.352 & 1.341 & 1.638 & 1.172 \\
\hline
\end{tabular}

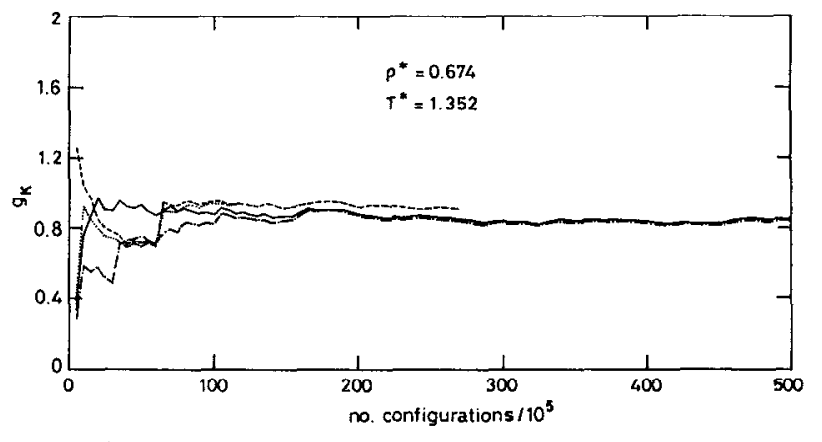

FIG. 2. Evolution of the Kirkwood factor $g_{K}$ along the simulation from the average $\left\langle M^{2}\right\rangle$ (solid and short dashed lines correspond to 500 and 864 particle samples, respectively) and from the fluctuation $\left\langle M^{2}\right\rangle-\langle M\rangle^{2}$ (dashed-dotted and dotted line correspond to 500 and 864 particle samples, respectively). Results for $258.15 \mathrm{~K}$.

of freedom in the polarizable fluid. The high-frequency dielectric constant $\epsilon_{\infty}$ can be calculated through the Clausius-Mosotti equation. With reaction field (RF) boundary conditions, the function $f(\epsilon)$ reduces to ${ }^{24}$

$$
f(\epsilon)=\frac{(\epsilon-1)\left(2 \epsilon_{\mathrm{RF}}+1\right)}{2 \epsilon_{\mathrm{RF}}+\epsilon} .
$$

In the present calculation we have used $\epsilon_{\mathrm{RF}}=7$, which is close to the experimental value of the dielectric constant for liquid $\mathrm{HCl}$. Moreover, according to Ref. 23, if the appropriate reaction field term is incorporated into the interaction potential and the corresponding formula for $f(\epsilon)$ is used, the results are independent of the particular choice of $\epsilon_{\mathrm{RF}}$.

\section{HNC AND RHNC EQUATIONS FOR HETERONUCLEAR TWO-CENTER LENNARD-JONES FLUIDS.}

The HNC theory for molecular fluids is well known and full details can be found in the standard literature. ${ }^{25}$ As for the reference hypernetted chain theory in its

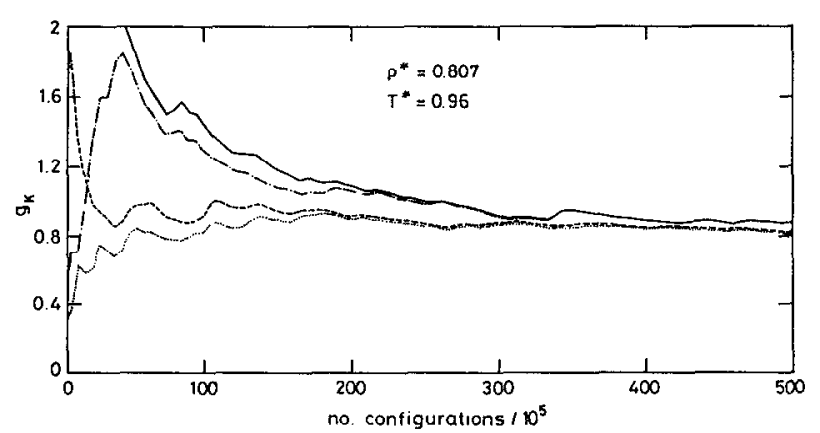

FIG. 3. Evolution of the Kirkwood factor $g_{X}$ along the simulation from the average $\left(M^{2}\right)$ (solid and short dashed lines correspond to 500 and 864 particle samples, respectively) and from the fluctuation $\left\langle M^{2}\right\rangle\langle-M\rangle^{2}$ (dashed-dotted and dotted line correspond to 500 and 864 particle samples, respectively). Results for $183.26 \mathrm{~K}$. 
RHNC-VM variety, here we merely recall its leading features. A more complete analysis can be found in the original papers. ${ }^{12,13}$

The HNC and RHNC-VM approximations stem from the Ornstein-Zernike equation coupled with a closure relation. In terms of the indirect correlation function $\gamma(12)$ $=h(12)-c(12)$, one has

$$
\gamma(12)=\frac{\rho}{4 \pi} \int c(13)[c(32)+\gamma(32)] d \mathrm{r}_{3} d \omega_{3}
$$

coupled with

$c(12)=\exp \left[-\beta u(12)+\gamma(12)-B_{0}(12)\right]-\gamma(12)-1$,

where in the HNC approximation $B_{0}(12)=0$ and in RHNC-VM it is the bridge function of a reference system, in this case a heteronuclear hard dumbbell. The latter function is determined using Verlet's approximation, ${ }^{15}$

$$
B_{0}(12)=\frac{1}{2} \frac{\gamma_{0}^{2}(12)}{1+a \gamma_{0}(12)}
$$

with $a$ given by

$$
a=1-\frac{1}{3} \rho^{*} d_{D}^{*^{3}}
$$

and

$$
\begin{aligned}
d_{D}= & \left\{\frac { 1 + \gamma } { 2 } \left[1+\frac{3 L^{*}\left(\gamma^{2}+1\right)}{2\left(\gamma^{3}+1\right)}-\frac{L^{* 3}}{\gamma^{3}+1}\right.\right. \\
& \left.\left.+\frac{3\left(\gamma^{2}-1\right)^{2}}{16 L^{*}\left(\gamma^{3}+1\right)}\right]\right\}^{1 / 3} d_{\mathrm{Cl}-\mathrm{Cl}-}^{*} .
\end{aligned}
$$

Here $L^{*}=l_{\mathrm{Cl}-\mathrm{H}} / \sigma_{\mathrm{Cl}-\mathrm{Cl}}$ is the reduced elongation and $\gamma=d_{\mathrm{H}-\mathrm{H}} / d_{\mathrm{Cl}-\mathrm{Cl}}$ is the ratio between the hard sphere diameters in the heteronuclear hard dumbbell.

Following Refs. 12 and 13, one might define $d_{\mathrm{Cl}-\mathrm{Cl}}^{*}=d_{\mathrm{Cl}-\mathrm{Cl}} / \sigma_{\mathrm{Cl}-\mathrm{Cl}}$ and $d_{\mathrm{H}-\mathrm{H}}^{*}=d_{\mathrm{H}-\mathrm{H}} / \sigma_{\mathrm{Cl}-\mathrm{Cl}}$ in terms of $k T / \epsilon_{\mathrm{Cl}-\mathrm{Cl}}, \rho \sigma_{\mathrm{Cl}-\mathrm{Cl}}$, and $k T / \epsilon_{\mathrm{H}-\mathrm{H}}, \rho \sigma_{\mathrm{H}-\mathrm{H}}$, but we have found that a more effective choice is made if $\gamma$ is taken as an adjustable parameter and $d_{\mathrm{Cl}-\mathrm{Cl}}$ is determined according the criterion defined in Ref. 13, namely

TABLE IV. Internal energy for liquid HCl. Monte Carlo vs RHNC results with three different reference systems described by the parameter $\gamma$ [see Eq. (10) in the text].

\begin{tabular}{cccccc}
\hline \hline$\rho^{*}$ & $T^{*}$ & MC & RHNC- $\gamma_{1}$ & RHNC- $\gamma_{2}$ & RHNC- $\gamma_{3}$ \\
\hline 0.80694 & 0.960 & -12.93 & -12.70 & -12.74 & -12.77 \\
0.67394 & 1.352 & -7.14 & -7.06 & -7.06 & -7.05 \\
\hline
\end{tabular}

TABLE V. Compressibility factor for liquid $\mathrm{HCl}$. Monte Carlo vs RHNC results with three different reference systems described by the parameter $\gamma$ [see Eq. (10) in the text].

\begin{tabular}{cccccc}
\hline \hline$\rho^{*}$ & $T^{*}$ & MC & RHNC- $\gamma_{1}$ & RHNC- $\gamma_{2}$ & RHNC- $\gamma_{3}$ \\
\hline 0.80694 & 0.960 & -1.57 & -1.43 & -2.68 & -4.46 \\
0.67394 & 1.352 & -0.65 & -0.63 & -1.19 & -1.70 \\
\hline \hline
\end{tabular}

$$
d_{\mathrm{Cl}-\mathrm{Cl}} / \sigma_{\mathrm{Cl}-\mathrm{Cl}}=\alpha\left(25-\log \frac{k T}{\epsilon_{\mathrm{Cl}-\mathrm{Cl}}}\right)\left(25+\log \rho \sigma_{\mathrm{Cl}-\mathrm{Cl}}^{3}\right),
$$

with $\alpha=1.62 \times 10^{-3}$. We have adjusted $\gamma$ to reproduce the Monte Carlo results at high density. The $\gamma$ parameter accounts for the geometry of the heteronuclear molecule while expression (11) incorporates the effects of the temperature and density on the reference system. We will see that this choice is sufficiently general and that the geometry parameter $\gamma$ adjusted at high density and low temperature yields even better results for higher $\mathrm{T}$ and lower $\rho$ values. In a work currently in progress, an empirical expression that determines the geometry of the reference system without resorting to the one-state fitting presented above is being investigated. ${ }^{18}$

Once the reference system is well characterized, fluid structure, thermodynamic quantities, and static dielectric constant are easily accessible using the standard procedures indicated in Refs. 12-14.

\section{RESULTS AND CONCLUSIONS}

In Tables IV and $\mathrm{V}$ we present results for the internal energy and compressibility factor obtained by computer simulation and evaluated in the RHNC approximation with various values for the parameter $\gamma$ of the reference system, which we have denoted by $\gamma_{1}, \gamma_{2}$, and $\gamma_{3}(0.6,0.7$, and 0.81 , respectively). The largest value corresponds to the quotient of the Lennard-Jones interaction range parameters, $\sigma_{\mathrm{H}-\mathrm{H}} / \sigma_{\mathrm{Cl}-\mathrm{Cl}}$, while $\gamma=0.7$ is the value obtained if Eq. (11) is used to compute both $d_{\mathrm{Cl}-\mathrm{Cl}}$ and $d_{\mathrm{H}-\mathrm{H}}$, using the corresponding Lennard-Jones parameters for each interaction. Finally, the value $\gamma=0.6$, which yields the results labeled RHNC- $\gamma_{1}$ in the tables, is determined by linear extrapolation from the RHNC results for the other $\gamma$ values so that the Lennard-Jones component of the internal energy at high density is reproduced. We see that this leads to a fair agreement for the total internal energy and compressibility, not only at high density, but also for the lower

TABLE VI. Excess internal energy in the HNC and RHNC approximations vs Monte Carlo results.

\begin{tabular}{ccccc}
\hline \hline$\rho^{*}$ & $T^{*}$ & MC & RHNC & HNC \\
\hline 0.80694 & 0.960 & -12.93 & -12.70 & -12.43 \\
0.67394 & 1.352 & -7.14 & -7.06 & -7.05 \\
\hline \hline
\end{tabular}


TABLE VII. Multipolar contributions to the internal energy. RHNC and HNC vs MC data.

\begin{tabular}{|c|c|c|c|c|c|c|c|c|c|c|}
\hline \multirow[b]{2}{*}{$\rho^{*}$} & \multirow[b]{2}{*}{$T^{*}$} & \multicolumn{3}{|c|}{$(\beta U / N)_{\mathrm{DD}}$} & \multicolumn{3}{|c|}{$(\beta U / N)_{\mathrm{DQ}}$} & \multicolumn{3}{|c|}{$(\beta U / N)_{Q Q}$} \\
\hline & & MC & RHNC & HNC & MC & RHNC & HNC & MC & RHNC & $\mathrm{HNC}$ \\
\hline 0.80694 & 0.960 & -0.85 & -0.84 & -0.83 & -2.27 & -2.19 & -2.29 & -2.17 & -1.97 & -2.13 \\
\hline 0.67394 & 1.352 & -0.51 & -0.50 & -0.53 & -1.16 & -1.13 & -1.19 & -1.04 & -0.98 & -1.03 \\
\hline
\end{tabular}

density and higher temperature case. The RHNC results given in the remainder of the paper will refer to those obtained with a $\gamma$ value of 0.6 .

We observe in Table VI that the RHNC closure used here gives slightly better internal energies than HNC, although HNC outperforms RHNC in just the multipolar contributions to the total internal energy, as can be seen in Table VII. This is a feature already observed in polar hard dumbbells ${ }^{12}$ and in polar hard spheres. ${ }^{26}$ The situation is completely different as far as the compressibility factor is concerned (see Table VIII). Here, the HNC fails badly, as one may expect. However, our empirically optimized RHNC leads to results in good accordance with the simulation data for the two states under consideration.

With regard to the orientational averages and dielectric constant, the situation is less satisfactory. In Table IX we summarize the results for the Kirkwood factor $\left(\left\langle M^{2}\right\rangle / N \mu^{2}\right)$. We see that the density (and temperature) dependence of this quantity predicted by the simulation is almost negligible, at least for the variation in thermodynamic state considered here. (Actually, the change in the Kirkwood factor is almost within the statistical uncertainty of the simulated values.) The HNC and RHNC results are hardly distinguishable from each other, as was also found for the polar hard dumbbell fluid in a previous work. ${ }^{10}$ In $^{2}$ addition, the theoretical results for this quantity, although in qualitative agreement with simulation, show a slightly stronger dependence on the thermodynamic state than do those of the Monte Carlo calculations. In Table X we compare the experimental dielectric constant of liquid hydrogen chloride $^{19}$ with the Monte Carlo and integral equation predictions. The most remarkable feature is the agreement between simulation and experiment. Although at the higher density the discrepancies are somewhat larger (as is the uncertainty of the experimental measures), the results may still be characterized as good. The theoretical predictions are purely qualitative, since both theories underestimate the Kirkwood factor for the higher density.

Finally, the microscopic structure (spatial and orientational) of the fluid is shown in Figs. 4-7, where we have plotted the center-to-center distribution function [radial coefficient $g_{000}$ of the spherical harmonic expansion of $g(12)]$ and the first three angular projections of the molecular pair distribution function, for the two thermodynamic states under study. We see that the HNC curves have their maxima and minima out of phase as usual and, in contrast, RHNC data, aside from minor deviations, reproduce the simulation structure in detail.

We can conclude that the results here introduced represent an encouraging step forward, showing the ability of
TABLE VIII. Compressibility factor in the HNC and RHNC approximations vs Monte Carlo results.

\begin{tabular}{ccccc}
\hline \hline$\rho^{*}$ & $T^{*}$ & MC & RHNC & HNC \\
\hline 0.80694 & 0.960 & -1.57 & -1.43 & 2.00 \\
0.67394 & 1.352 & -0.65 & -0.63 & 0.72 \\
\hline \hline
\end{tabular}

TABLE IX. Kirkwood factor in the HNC and RHNC approximations vs Monte Carlo results.

\begin{tabular}{ccccc}
\hline$\rho^{*}$ & $T^{*}$ & MC & RHNC & HNC \\
\hline 0.80694 & 0.960 & 0.87 & 0.79 & 0.78 \\
0.67394 & 1.352 & 0.84 & 0.89 & 0.88 \\
\hline \hline
\end{tabular}

TABLE X. Dielectric constant for the polarizable model of $\mathrm{HCl}$. HNC, RHNC, and Monte Carlo reaction field calculations vs experimental data.

\begin{tabular}{cccccc}
\hline \hline$\rho^{*}$ & $T^{*}$ & Expt. & MC & RHNC & HNC \\
\hline 0.80694 & 0.960 & $10.6-11.7$ & 10.40 & 9.44 & 9.25 \\
0.67394 & 1.352 & 6.32 & 6.31 & 6.58 & 6.48 \\
\hline \hline
\end{tabular}

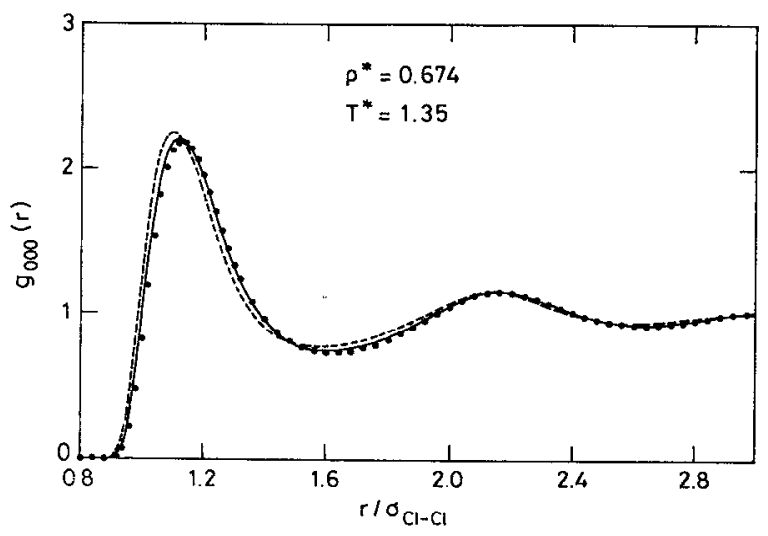

FIG. 4. Center-to-center distribution function for $\mathrm{HCl}$ at $258.15 \mathrm{~K}$ and $0.98 \mathrm{~g} / \mathrm{cm}^{3}$. The solid line corresponds to the empirically optimized RHNC equation, HNC results are denoted by the dashed line and solid circles are Monte Carlo results. 


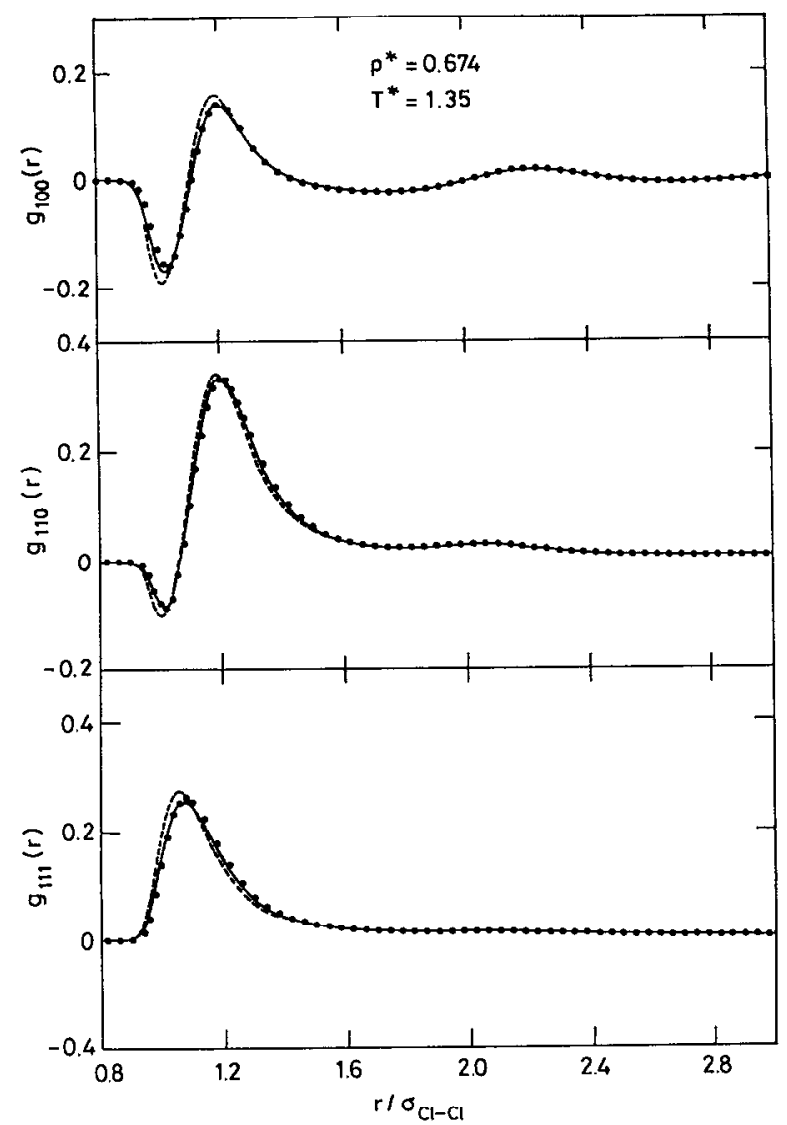

FIG. 5. First angular projections of the molecular distribution function for $\mathrm{HCl}$ at $258.15 \mathrm{~K}$ and $0.98 \mathrm{~g} / \mathrm{cm}^{3}$. The solid line corresponds to the empirically optimized RHNC equation, HNC results are denoted by the dashed line and solid circles are Monte Carlo results.

theoretical tools to investigate systems with realistic interactions. Moreover, the use of effective polar fluids to incorporate the effects of polarizability has proven successful in this model of a molecular fluid, as was the SCMF theory for the spherically shaped model of water studied by Patey, Levesque, and Weis. ${ }^{7}$ The fact that the quality of the re-

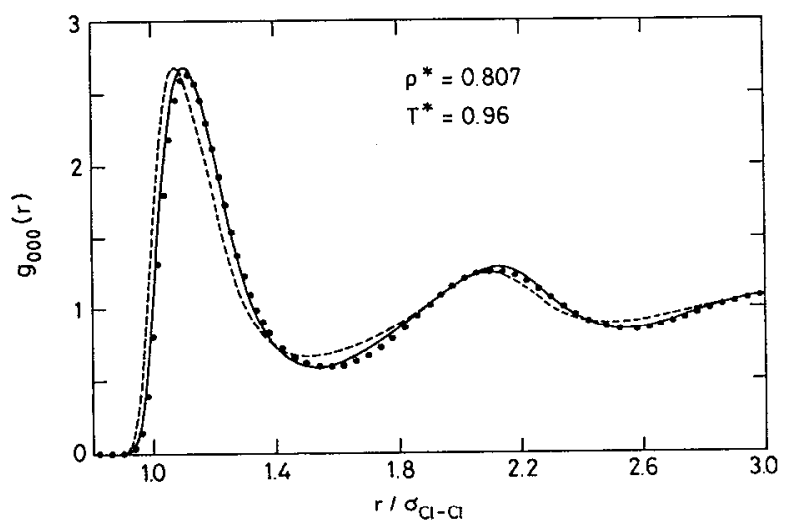

FIG. 6. Center-to-center distribution function for $\mathrm{HCl}$ at $183.26 \mathrm{~K}$ and $1.17 \mathrm{~g} / \mathrm{cm}^{3}$. The solid line corresponds to the empirically optimized RHNC equation, HNC results are denoted by the dashed line and solid circles are Monte Carlo results.

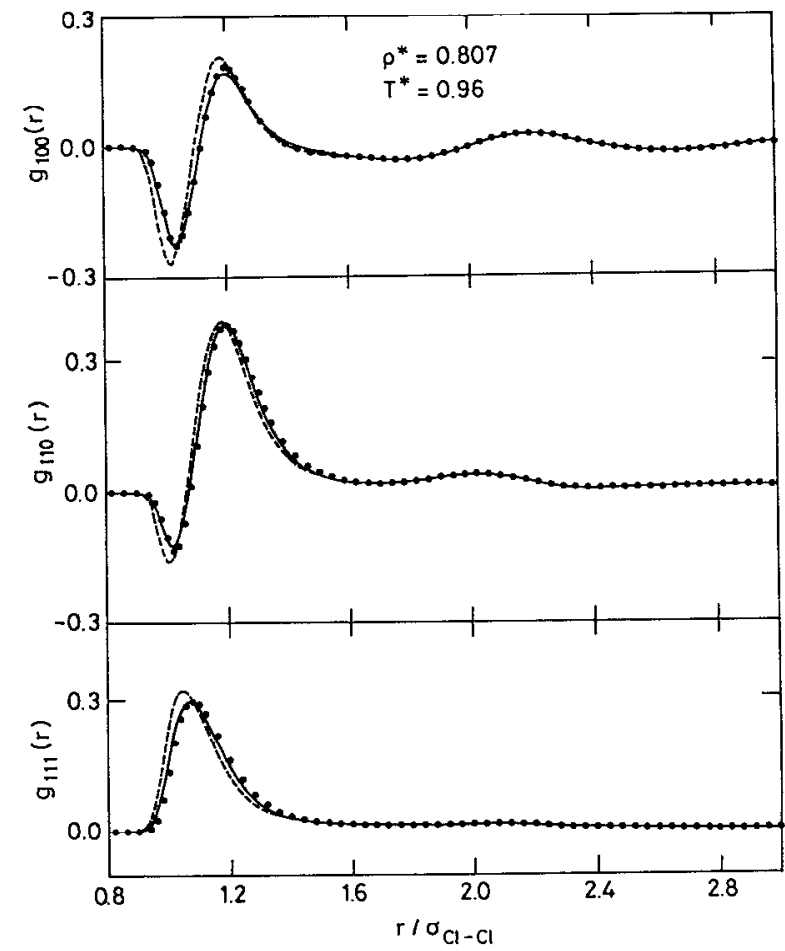

FIG. 7. First angular projections of the molecular distribution function for $\mathrm{HCl}$ at $183.26 \mathrm{~K}$ and $1.17 \mathrm{~g} / \mathrm{cm}^{3}$. The solid line corresponds to the empirically optimized RHNC equation, HNC results are denoted by the dashed line and solid circles are Monte Carlo results.

sults holds up for two thermodynamic states indicates, in our opinion, that the validity of the approach is fully justified, at least for the low values of polarizability corresponding to $\mathrm{HCl}$.

\section{ACKNOWLEDGMENTS}

NFR (Norges Forskningsråd) is acknowledged for the support provided to cover computer time expenses at the CRAY Y-MP in SINTEF (Trondheim, Norway). We also acknowledge the Centro Técnico de Informática del CSIC and the Centro de Supercomputación de Galicia (CESGA) for generous access to their computing facilities. This research was financially supported by the Spanish Dirección General de Investigación Científica y Técnica (DGICYT) under Grant No. PB91-0110.

${ }^{\text {I }}$ J. G. Powles, W. A. B. Evans, E. McGrath, K. E. Gubbins, and S. Murad, Mol. Phys. 38, 893 (1979).

${ }^{2}$ S. Murad, K. E. Gubbins, and J. G. Powles, Mol. Phys. 40, 253 (1980).

${ }^{3}$ W. A. B. Evans and J. G. Powles, Mol. Phys. 45, 695 (1982).

${ }^{4}$ I. R. McDonald, S. F. O'Shea, D. G. Bounds, and M. L. Klein, J. Chem. Phys. 72, 5710 (1980).

${ }^{5}$ E. Lomba, A. Fernández, C. Martín, and M. Lombardero, Phys. Scr. T38, 79 (1991).

${ }^{6}$ J. S. Hoye and E. Lomba, Mol. Phys. (in press).

${ }^{7}$ G. N. Patey, D. Levesque, and J. J. Weis, Mol. Phys. 57, 337 (1986).

${ }^{8}$ J. M. Caillol, D. Levesque, J. J. Weis, J. S. Perkins, and G. N. Patcy, Mol. Phys. 62, 1225 (1987).

${ }^{9}$ F. Lado, M. Lombardero, E. Enciso, S. Lago, and J. L. F. Abascal, J. Chem. Phys. 85, 2916 (1986).

${ }^{10}$ E. Lomba, M. Lombardero, and J. L. F. Abascal, J. Chem. Phys. 90, 7330 (1989). 
"A. Nichols III and D. Calef, Mol. Phys. 71, 269 (1989).

${ }^{12}$ E. Lomba, C. Martín, M. Lombardero, and J. A. Anta, J. Chem. Phys. 96, 6132 (1992).

${ }^{13}$ M. Lombardero, C. Martín, and E. Lomba, J. Chem. Phys. 97, 2724 (1992).

${ }^{14}$ C. Martín, M. Lombardero, and E. Lomba, J. Chem. Phys. 98, 6465 (1992).

${ }^{15}$ L. Verlet, Mol. Phys. 41, 183 (1980).

${ }^{16}$ S. Labik, A. Malijevsky, and W. R. Smith, Mol. Phys. 73, 87 (1991).

${ }^{17}$ S. Labik, A. Malijevsky, and W. R. Smith, Mol. Phys. 73, 495 (1991).

${ }^{18} \mathrm{M}$. Lombardero, C. Martín, and E. Lomba, Mol. Phys. (in press).

${ }^{19}$ G. Glockler and R. E. Peck, J. Chem. Phys. 4, 658 (1936); R. W.
Swanson and R. H. Cole, J. Chem. Phys. 22, 284 (1954).

${ }^{20}$ E. Lomba, M. Lombardero, and J. L. F. Abascal, Mol. Phys. 68, 1067 (1989).

${ }^{21}$ X. Ni and R. M. Fine, J. Phys. Chem. 96, 2718 (1992).

${ }^{22}$ J. A. Barker and R. O. Watts, Chem. Phys. Lett. 3, 144 (1969).

${ }^{23}$ G. N. Patey, D. Levesque, and J. J. Weis, Mol. Phys. 45, 733 (1982).

${ }^{24}$ S.W. de Leeuw, J. W. Perram, and E. R. Smith, Ann. Rev. Phys. Chem. 37,245 (1986).

${ }^{25}$ C. G. Gray and K. E. Gubbins, Theory of Molecular Fluids (Clarendon, Oxford, 1984).

${ }^{26}$ E. Lomba, C. Martín, and M. Lombardero, Mol. Phys. 77, 1005 (1992). 